PROCEEDINGS OF THE

AMERICAN MATHEMATICAL SOCIETY

Volume 138, Number 7, July 2010, Pages 2289-2299

S 0002-9939(10)10300-1

Article electronically published on February 24, 2010

\title{
ZEROS OF THE EISENSTEIN SERIES $E_{2}$
}

\author{
ABDELKRIM EL BASRAOUI AND ABDELLAH SEBBAR
}

(Communicated by Keno Ono)

\begin{abstract}
In this paper we investigate the zeros of the Eisenstein series $E_{2}$. In particular, we prove that $E_{2}$ has infinitely many $\mathrm{SL}_{2}(\mathbb{Z})$-inequivalent zeros in the upper half-plane $\mathfrak{H}$, yet none in the standard fundamental $\mathfrak{F}$. Furthermore, we go on to investigate other fundamental regions in the upper half-plane $\mathfrak{H}$ for which there do or do not exist zeros of $E_{2}$. We establish infinitely many such regions containing a zero of $E_{2}$ and infinitely many which do not.
\end{abstract}

\section{INTRODUCTION}

Let $\mathfrak{H}=\{\tau \in \mathbb{C}, \operatorname{Im}(\tau)>0\}$ be the upper half-plane. The Eisenstein series are defined for every even integer $k \geq 2$ and $\tau \in \mathfrak{H}$ by

$$
\begin{aligned}
E_{k}(\tau) & =1-\frac{2 k}{B_{k}} \sum_{n=1}^{\infty} \sigma_{k-1}(n) q^{n} \\
& =1-\frac{2 k}{B_{k}} \sum_{n=1}^{\infty} \frac{n^{k-1} q^{n}}{1-q^{n}}, \quad q=e^{2 \pi i \tau} .
\end{aligned}
$$

Here $B_{k}$ is the $k$-th Bernoulli number and $\sigma_{k}(n)=\sum_{d \mid n} d^{k}$.

These series play an important role in the theory of modular forms and quasimodular forms. They have been the topic of extensive investigation for a long time from various points of view. For instance, from the analytic point of view, the study of the zeros of $E_{k}(z), k \geq 4$, has been carried out by several authors. In 1963 , K. Wohlfahrt proved in [6] that the zeros of $E_{k}, 4 \leq k \leq 26$, are simple and lie in the arc of the unit circle $\left\{z=e^{i \theta}: \pi / 2 \leq \theta \leq 3 \pi / 2\right\}$ in the fundamental domain $\mathfrak{F}=\{\tau \in \mathfrak{H},|\tau| \geq 1$ and $|\operatorname{Re}(\tau)| \leq 1 / 2\}$ of the modular group $\mathrm{SL}_{2}(\mathbb{Z})$. He also conjectured that this holds for all $k \geq 4$. In 1970, F.K.C. Rankin and H.P.F. Swinnerton-Dyer [5] proved Wohlfahrt's conjecture. In 1982, R.A. Rankin [4] generalized their result to a certain class of Poincaré series. However, nothing has been proven for the Eisenstein series $E_{2}$, which is important in many fields. In fact, even whether it has a finite or an infinite number of zeros has not been known.

In this paper, we prove that there are infinitely many non-equivalent zeros of $E_{2}$ in $\mathfrak{H}$. In fact, since $E_{2}$ is not exactly a modular form but rather a quasi-modular form, two zeros $\tau_{0}$ and $\tau_{1}$ of $E_{2}$ are $\mathrm{SL}_{2}(\mathbb{Z})$-equivalent, that is $\tau_{1}=\gamma \cdot \tau_{0}$ for $\gamma \in \mathrm{SL}_{2}(\mathbb{Z})$ if and only if $\tau_{1}=\tau_{0}+n$ for an integer $n$. Thus, we restrict our investigation to the half-strip $\mathfrak{S}=\left\{\tau \in \mathfrak{H},-\frac{1}{2}<\operatorname{Re}(\tau) \leq \frac{1}{2}\right\}$, in which we prove

Received by the editors April 21, 2009, and, in revised form, October 3, 2009.

2010 Mathematics Subject Classification. Primary 11F11.

(C)2010 American Mathematical Society Reverts to public domain 28 years from publication 
that there are infinitely many zeros for $E_{2}$. Moreover, these zeros present a strange distribution in $\mathfrak{S}$. More precisely, the fundamental domain $\mathfrak{F}$ and infinitely many of its conjugates within $\mathfrak{S}$ contain no zero of $E_{2}$, while there are infinitely many conjugates of $\mathfrak{F}$ which contain zeros of $E_{2}$.

\section{EISENSTEIN SERIES: SOME PROPERTIES}

The most familiar Eisenstein series are

$$
\begin{array}{lll}
E_{2}(\tau)=1-24 & \sum_{n=1}^{\infty} \sigma_{1}(n) q^{n}, \\
E_{4}(\tau)=1+240 & \sum_{n=1}^{\infty} \sigma_{3}(n) q^{n}, \\
E_{6}(\tau)=1-504 \sum_{n=1}^{\infty} \sigma_{5}(n) q^{n} .
\end{array}
$$

The series $E_{4}$ and $E_{6}$ are, respectively, modular forms of weight 4 and 6 . However, the Eisenstein series $E_{2}$ is not a modular form. In fact, it transforms under the action of the modular group as follows (see [3]).

Proposition 2.1. For $\alpha=\left(\begin{array}{ll}a & b \\ c & d\end{array}\right) \in S L_{2}(\mathbb{Z})$, we have

$$
E_{2}(\alpha \cdot \tau)=(c \tau+d)^{2} E_{2}(\tau)+\frac{6 c}{\pi i}(c \tau+d)
$$

where

$$
\alpha \cdot \tau=\frac{a \tau+b}{c \tau+d} .
$$

This proposition can be proved using the fact that $E_{2}$ is the logarithmic derivative of the modular discriminant $\Delta=\frac{1}{1728}\left(E_{4}^{3}-E_{6}^{2}\right)$ of weight 12 , the derivation being $\frac{1}{2 \pi i} \frac{d}{d \tau}$.

These three functions were especially studied by Ramanujan [2, who proved that they satisfy the following differential equations:

$$
\begin{aligned}
\frac{1}{2 \pi i} \frac{d E_{2}}{d \tau} & =\frac{1}{12}\left(E_{2}^{2}-E_{4}\right), \\
\frac{1}{2 \pi i} \frac{d E_{4}}{d \tau} & =\frac{1}{3}\left(E_{2} E_{4}-E_{6}\right), \\
\frac{1}{2 \pi i} \frac{d E_{6}}{d \tau} & =\frac{1}{2}\left(E_{2} E_{6}-E_{4}^{2}\right) .
\end{aligned}
$$

Thus the graded ring $\mathbb{C}\left[E_{2}, E_{4}, E_{6}\right]$ is closed under the differential operator $\frac{d}{d \tau}$. It is known that the space of all modular forms is exactly the graded ring $\mathbb{C}\left[E_{4}, E_{6}\right]$. We shall at this stage give some special values of $E_{2}$ at $i$ and at the cubic root of unity $\rho=\frac{-1+i \sqrt{3}}{2}$ :

$$
\begin{aligned}
E_{2}(i) & =\frac{3}{\pi}, \\
E_{2}(\rho) & =\frac{2 \sqrt{3}}{\pi} .
\end{aligned}
$$

This follows from the transformation formula for $E_{2}$ together with the appropriate transformations that fix $i$ and $\rho$. 


\section{Zeros of the Eisenstein SERIES $E_{2}$}

In this section we prove that the series $E_{2}$ has infinitely many zeros, a fact that has not been known before. Set $S=\left(\begin{array}{rr}0 & -1 \\ 1 & 0\end{array}\right)$ and $S_{n}=\left(\begin{array}{cc}1 & 0 \\ n & 1\end{array}\right)$ for positive integers $n$.

Proposition 3.1. The Eisenstein series $E_{2}$ has a zero $\tau_{0}$, on the imaginary axis and a zero $\tau_{1}$ on the axis $\operatorname{Re}(z)=\frac{1}{2}$.

Proof. It is clear that for $\tau=i y$, the series $E_{2}(\tau)$ is real and increasing on $(0, \infty)$. Meanwhile, $\lim _{y \rightarrow 0} E_{2}(i y)=-\infty$ and $\lim _{y \rightarrow \infty} E_{2}(i y)=1$. It follows that $E_{2}$ has a unique zero, say $\tau_{0}$, on the purely imaginary axis.

Similarly, $E_{2}(\tau)$ is real for $\tau=1 / 2+i y, y>0$. Furthermore, we have

$$
\lim _{y \mapsto 0} E_{2}\left(\frac{1}{2}+i y\right)=-\infty
$$

Indeed, for $\alpha=S_{2}^{-1}=\left(\begin{array}{rr}1 & 0 \\ -2 & 1\end{array}\right)$ we have

$$
E_{2}\left(\frac{1}{2}+i y\right)=-\frac{1}{y^{2}}\left(\frac{1}{4} E_{2}\left(-\frac{1}{2}+\frac{i}{4 y}\right)-\frac{6 y}{\pi}\right) .
$$

This gives the desired limit since $E_{2}\left(-\frac{1}{2}+\frac{i}{4 y}\right)$ tends to 1 as $y$ tends to 0 . Combining this with the fact that $E_{2}(\rho)=E_{2}(\rho+1)=\frac{2 \sqrt{3}}{\pi}$ yields the existence of a zero $\tau_{1}$ of real part $1 / 2$ and whose imaginary part is less than $\sqrt{3} / 2$. Here again we used the transformation formula in Proposition 2.1 with $\alpha=\left(\begin{array}{ll}1 & 1 \\ 0 & 1\end{array}\right)$.

As for the location of these two zeros, and taking into account the special value of $E_{2}$ at $i$ and $\rho$ given respectively by (9) and (10), we have

Proposition 3.2. The zeros $\tau_{0}$ and $\tau_{1}$ are contained respectively in the fundamental domains $S \mathfrak{F}, S_{2} \mathfrak{F}$.

It is worth mentioning that numerical values of these two zeros appear in [1], where they are studied as equilibrium points of Green's functions.

Unlike the case of modular forms, the set of zeros of $E_{2}$ is not invariant under every conjugation by elements of $\mathrm{SL}_{2}(\mathbb{Z})$. In fact we have

Proposition 3.3. Two zeros of $E_{2}$ are equivalent if and only if one is a translate of the other by an integer.

Proof. Suppose that $z_{1}, z_{2}$ are any two zeros of $E_{2}$ in the half-plane $\mathfrak{H}$ that are equivalent modulo $\mathrm{SL}_{2}(\mathbb{Z})$. Say, $z_{1}=\alpha \cdot z_{2}, \alpha=\left(\begin{array}{ll}a & b \\ c & d\end{array}\right)$. Then, by the transformation formula for $E_{2}$ in Proposition 2.1, we have

$$
E_{2}\left(z_{1}\right)=0=E_{2}\left(\alpha \cdot z_{2}\right)=\left(c z_{2}+d\right)^{2} E_{2}\left(z_{2}\right)+\frac{6 c}{\pi i}\left(c z_{2}+d\right)=\frac{6 c}{\pi i}\left(c z_{2}+d\right),
$$

which is possible only when $c=0$, and in this case we have $a=d= \pm 1$; that is, $\alpha$ is a translation. The converse follows from the invariance of $E_{2}$ under translation. 
As a consequence we have

Corollary 3.4. No two distinct zeros of $E_{2}$ in the half-strip $\mathfrak{S}$ are equivalent modulo the modular group $S L_{2}(\mathbb{Z})$.

We now state the main results of this section.

Theorem 3.5. The Eisenstein series $E_{2}$ has infinitely many zeros in the half-strip $\mathfrak{S}=\left\{\tau \in \mathfrak{H},-\frac{1}{2}<\operatorname{Re}(\tau) \leq \frac{1}{2}\right\}$

Proof. Let $\tau_{0}$ be the unique zero of $E_{2}$ on the imaginary axis. Let $\alpha=\left(\begin{array}{cc}t & u \\ v & w\end{array}\right) \in$ $\mathrm{SL}_{2}(\mathbb{Z})$, where $t v \neq 0$. Then, by Equation (5), we have

$$
E_{2}\left(\tau_{0}\right)=0=E_{2}\left(\alpha^{-1} \alpha \cdot \tau_{0}\right)=\left(-v\left(\alpha \cdot \tau_{0}\right)+t\right)^{2} E_{2}\left(\alpha \cdot \tau_{0}\right)-\frac{6 v}{\pi i}\left(-v\left(\alpha \cdot \tau_{0}\right)+t\right) .
$$

It follows that

$$
\left(-v\left(\alpha \cdot \tau_{0}\right)+t\right) E_{2}\left(\alpha \cdot \tau_{0}\right)=\frac{6 v}{\pi i},
$$

which is equivalent to saying that

$$
\frac{E_{2}\left(\alpha \cdot \tau_{0}\right)}{\left(\alpha \cdot \tau_{0}\right) E_{2}\left(\alpha \cdot \tau_{0}\right)+\frac{6}{\pi i}}=\frac{v}{t} .
$$

This means that the map $f(z)$ defined by

$$
f(z)=\frac{E_{2}(z)}{\left(z E_{2}(z)+\frac{6}{\pi i}\right)}
$$

carries $\alpha \cdot \tau_{0}$ onto $r_{0}=v / t$, and thus it maps any open neighborhood $D_{0}$ of $\alpha \cdot \tau_{0}$, which we choose in the interior of the fundamental domain $\alpha S \mathfrak{F}$ and on which it is holomorphic, onto an open neighborhood $U_{0}$ of $r_{0}$. Let $r_{1}=a_{1} / b_{1}$ be a reduced fraction in $\mathbb{Q} \cap U_{0} \backslash\left\{r_{0}\right\}$. Then there exists $z_{1} \in D_{0} \backslash\left\{\alpha \cdot \tau_{0}\right\}$ such that $f\left(z_{1}\right)=a_{1} / b_{1}$. Therefore,

$$
\left(-a_{1} z_{1}+b_{1}\right) E_{2}\left(z_{1}\right)=\frac{6 a_{1}}{\pi i}
$$

Choose $c_{1}, d_{1} \in \mathbb{Z}$ such that $b_{1} d_{1}-a_{1} c_{1}=1$. Then

$$
\gamma_{1}:=\left(\begin{array}{cc}
d_{1} & -c_{1} \\
-a_{1} & b_{1}
\end{array}\right) \in \mathrm{SL}_{2}(\mathbb{Z}) .
$$

If we set $\tau_{1}=\gamma_{1} \cdot z_{1}$, then, using (5) and (11), we have $E_{2}\left(\tau_{1}\right)=0$. Moreover, $\tau_{1}$ is not equivalent to $\tau_{0}$ modulo $\mathrm{SL}_{2}(\mathbb{Z})$; otherwise we would have, according to Proposition 3.3. that $\tau_{0}:=T^{n} \gamma_{1} \cdot z_{1}$ for some $n \in \mathbb{Z}$ with $T=\left(\begin{array}{ll}1 & 1 \\ 0 & 1\end{array}\right)$. Since $z_{1} \in \alpha S \mathfrak{F}$, write $z_{1}=\alpha \cdot z_{1}^{\prime}$ for some $z_{1}^{\prime} \in S \mathfrak{F}$. We have $\tau_{0}=T^{n} \gamma_{1} \alpha \cdot z_{1}^{\prime}$ with $\tau_{0}$ and $z_{1}^{\prime}$ being in the fundamental domain $S \mathfrak{F}$. Therefore, $T^{n} \gamma_{1} \alpha=1$, and hence $\tau_{0}=z_{1}^{\prime}$ and $\alpha \cdot \tau_{0}=z_{1}$, a contradiction since we have chosen $z_{1} \in D_{0} \backslash\left\{\alpha \tau_{0}\right\}$. Thus $\tau_{1}$ is a zero of $E_{2}$ that is not equivalent to $\tau_{0}$.

It remains to show that two distinct rational numbers lead to two distinct zeros of $E_{2}$. Let $r_{2}=a_{2} / b_{2}$ be a rational number in $U_{0} \backslash\left\{r_{0}, r_{1}\right\}$. In the same way we construct a zero of $E_{2}, \tau_{2}=\gamma_{2} \cdot z_{2}$, that is not equivalent to $\tau_{0}$ modulo $\mathrm{SL}_{2}(\mathbb{Z})$, with $z_{2} \in \alpha S \mathfrak{F}$. Then $\tau_{2}$ is not equivalent to $\tau_{1}$ modulo $\mathrm{SL}_{2}(\mathbb{Z})$. Indeed if $\tau_{1}=T^{m} \cdot \tau_{2}$ for some $m \in \mathbb{Z}$, then $\gamma_{1} \alpha \cdot z_{1}^{\prime}=T^{m} \gamma_{2} \alpha \cdot z_{2}^{\prime}$ with $z_{1}^{\prime}$ and $z_{2}^{\prime}$ being in the same fundamental domain $S \mathfrak{F}$. It follows that $\gamma_{1} \alpha=T^{m} \gamma_{2} \alpha$, and consequently $r_{1}=r_{2}$. 
This contradicts our choice of $r_{2}$. Hence, $\tau_{2}$ is another zero of $E_{2}$ that is not equivalent to either $\tau_{0}$ or $\tau_{1}$. Finally, since the open set $U_{0}$ contains infinitely many rational numbers, we deduce that $E_{2}$ has infinitely many zeros in the half-strip $\mathfrak{S .}$

Since $E_{2}$ is the logarithmic derivative of the discriminant $\Delta$, from the above theorem we deduce

Corollary 3.6. The discriminant $\Delta$ has infinitely many critical points.

We now look at the multiplicity of the zeros of $E_{2}$.

Theorem 3.7. The zeros of the Eisenstein series $E_{2}$ are all simple.

Proof. Let $z_{0}$ be a zero of $E_{2}$. By (6), we have

$$
\frac{1}{2 \pi i} \frac{d E_{2}\left(z_{0}\right)}{d \tau}=\frac{1}{12}\left(E_{2}\left(z_{0}\right)^{2}-E_{4}\left(z_{0}\right)\right)=\frac{-1}{12} E_{4}\left(z_{0}\right) .
$$

Therefore, to prove that this zero is simple, it suffices to show that $E_{4}\left(z_{0}\right) \neq 0$. It is known that $E_{4}$ has all its zeros at $\rho=\frac{-1+i \sqrt{3}}{2}$ and its conjugates modulo $\mathrm{SL}_{2}(\mathbb{Z})$ (see for instance [3]). Thus, it is enough to show that $E_{2}(\alpha \cdot \rho) \neq 0$ for all $\alpha \in \mathrm{SL}_{2}(\mathbb{Z})$. Using (5) and (10), we have for $\alpha=\left(\begin{array}{ll}a & b \\ c & d\end{array}\right) \in \mathrm{SL}_{2}(\mathbb{Z})$ :

$$
E_{2}(\alpha \cdot \rho)=(c \rho+d)^{2} \frac{2 \sqrt{3}}{\pi}+\frac{6 c}{\pi i}(c \rho+d)=\frac{2 \sqrt{3}}{\pi}\left(c^{2}-c d+d^{2}\right),
$$

which does not vanish unless $c=d=0$, which is not the case since $a d-b c=1$. This shows that $E_{2}$ does not vanish on the orbit of $\rho$ and that consequently $E_{4}$ and $E_{2}$ have no common zeros.

\section{Distribution of the Zeros of $E_{2}$}

In this section, we will show that there are infinitely many fundamental regions within the half-strip $\mathfrak{S}$ that contain zeros of $E_{2}$, and we will also show that there are infinitely many such regions that do not contain any zero of $E_{2}$.

Theorem 4.1. There is a positive integer $c_{0}$ such that for all integers $c \geq c_{0}$, there is a fundamental domain with a vertex at $1 / c$ containing a zero of $E_{2}$.

Proof. Let $\tau_{0}$ again denote the unique zero of $E_{2}$ on the imaginary axis, and let $\alpha=\left(\begin{array}{ll}t & u \\ v & w\end{array}\right) \in \mathrm{SL}_{2}(\mathbb{Z})$, so that $t v \neq 0$. As in the proof of Theorem 3.5 the map

$$
f(z)=\frac{E_{2}(z)}{\left(z E_{2}(z)+\frac{6}{\pi i}\right)}
$$

maps any neighborhood of $\alpha \cdot \tau_{0}$ onto a neighborhood of $v / t$. In particular, $f$ maps a neighborhood $D_{0}$ of $S_{1} \tau_{0}$, chosen to be in the interior of $S_{1} S \mathfrak{F}$, onto a neighborhood $U_{0}$ of 1 (recall that $S_{1}=\left(\begin{array}{ll}1 & 0 \\ 1 & 1\end{array}\right)$ ). There exists a positive integer $c_{0}$ such that for all $c \geq c_{0}, 1+1 / c \in U_{0}$. For each $c \geq c_{0}$, let $z_{c} \in D_{0}$ be such that $f\left(z_{c}\right)=1+1 / c$. Therefore, if $\gamma_{c}=\left(\begin{array}{cc}-1 & 1 \\ -1-c c & c\end{array}\right)$, then, as in the proof of Theorem 3.5 $\gamma_{c}{ }^{-1} \cdot z_{c}$ is a zero $E_{2}$ belonging to $\gamma_{c}{ }^{-1} S_{1} S \mathfrak{F}$. If we set $S_{c}=\gamma_{c}{ }^{-1} S_{1} S=\left(\begin{array}{ll}1 & 0 \\ c & 1\end{array}\right)$ for $c \geq c_{0}$, then we have constructed a zero of $E_{2}$ in the fundamental domain $S_{c} \mathfrak{F}$ which has a vertex at the cusp $1 / c$. 
Remark 4.1.

- Thanks to Proposition 3.3, the above theorem can be extended to include the cusps 0 and $1 / 2$.

- An immediate consequence of this theorem is again the infiniteness of the number of zeros of the Eisenstein series $E_{2}$. Furthermore, it follows from Corollary 3.4 that all these zeros are inequivalent modulo $\mathrm{SL}_{2}(\mathbb{Z})$, as all these fundamental domains are contained in the half-strip $\mathfrak{S}$.

We now focus on the fundamental domains that contain no zeros of $E_{2}$.

Proposition 4.2. The Eisenstein series $E_{2}$ has no zeros in the fundamental domain $\mathfrak{F}$ of $S L_{2}(\mathbb{Z})$.

Proof. Let $\tau_{0}=i y_{0}$ be the unique zero of $E_{2}$ on the imaginary axis. Using the transformation formula for $E_{2}$, we have

$$
0<E_{2}\left(-1 / \tau_{0}\right)=\frac{6}{\pi} y_{0}<1
$$

This follows from the fact that $\operatorname{Im}\left(\tau_{0}\right)<1$ (since $\left.\tau_{0} \in S \mathfrak{F}\right)$ and thus $\operatorname{Im}\left(-1 / \tau_{0}\right)>$ $\operatorname{Im}\left(\tau_{0}\right)$, and the fact that $E_{2}$ is strictly increasing on the imaginary axis with the value 0 at $\tau_{0}$ and the value 1 at $i \infty$. Therefore

$$
y_{0}<\frac{\pi}{6} \text {. }
$$

If $\tau=x+i y \in \mathfrak{F}$ is a zero of $E_{2}$, then $\operatorname{Im}(\tau)>\sqrt{3} / 2>\pi / 6>y_{0}$ and therefore

$$
\frac{1}{24}\left|1-E_{2}(\tau)\right|=\left|\sum_{n=1}^{\infty} \sigma_{1}(n) e^{2 \pi i n \tau}\right| \leq \sum_{n=1}^{\infty} \sigma_{1}(n) e^{-2 \pi n y}<\sum_{n=1}^{\infty} \sigma_{1}(n) e^{-2 \pi n y_{0}} .
$$

The latter sum is simply $1 / 24\left(1-E_{2}\left(\tau_{0}\right)\right)=1 / 24$. Therefore

$$
\frac{1}{24}\left|1-E_{2}(\tau)\right|<\frac{1}{24}
$$

Hence $E_{2}(\tau)$ cannot be 0 if $\tau \in \mathfrak{F}$.

In the above proof we have used the inequality $\sqrt{3} / 2>\pi / 6$, which is obvious numerically but is a consequence of a simpler inequality such as $\pi<4$. In what follows we will rely on another inequality which is also numerically obvious:

$$
e^{-\pi \sqrt{3}}<\frac{1}{200}
$$

It simply says that $0.00433<0.005$.

We will now investigate more fundamental domains that do not contain any zeros of $E_{2}$. For a fixed integer $c \geq 2$ we again set $S_{c}=\left(\begin{array}{ll}1 & 0 \\ c & 1\end{array}\right)$ and $S_{b, d}(c)=$ $\left(\begin{array}{ll}1 & b \\ c & d\end{array}\right) \in \mathrm{SL}_{2}(\mathbb{Z}), b, d \in \mathbb{Z}$, and $\delta_{b}=\left(\begin{array}{cc}0 & -1 \\ 1 & b\end{array}\right) \in \mathrm{SL}_{2}(\mathbb{Z}), b \in \mathbb{Z}$. The fundamental domain $S_{b, d}(c) \mathfrak{F}$ has a vertex at the cusp $1 / c$, as does $S_{c} \mathfrak{F}$. Also $\delta_{b} \mathfrak{F}$ has a vertex at the cusp 0 , as does $S \mathfrak{F}$.

Let us examine more closely the fundamental domain $S_{c} \mathfrak{F}$. Its vertices are

$$
\frac{1}{c}, \quad S_{c} \cdot \rho=\frac{c-\frac{1}{2}+i \frac{\sqrt{3}}{2}}{c^{2}-c+1}, \quad S_{c} \cdot(\rho+1)=\frac{c+\frac{1}{2}+i \frac{\sqrt{3}}{2}}{c^{2}+c+1} .
$$

It is clear that $\operatorname{Im}\left(S_{c} \cdot \rho\right)>\operatorname{Im} S_{c} \cdot(\rho+1)$ and $\operatorname{Re} S_{c} \cdot \rho>1 / c>\operatorname{Re} S_{c} \cdot(\rho+1)$. Thus 


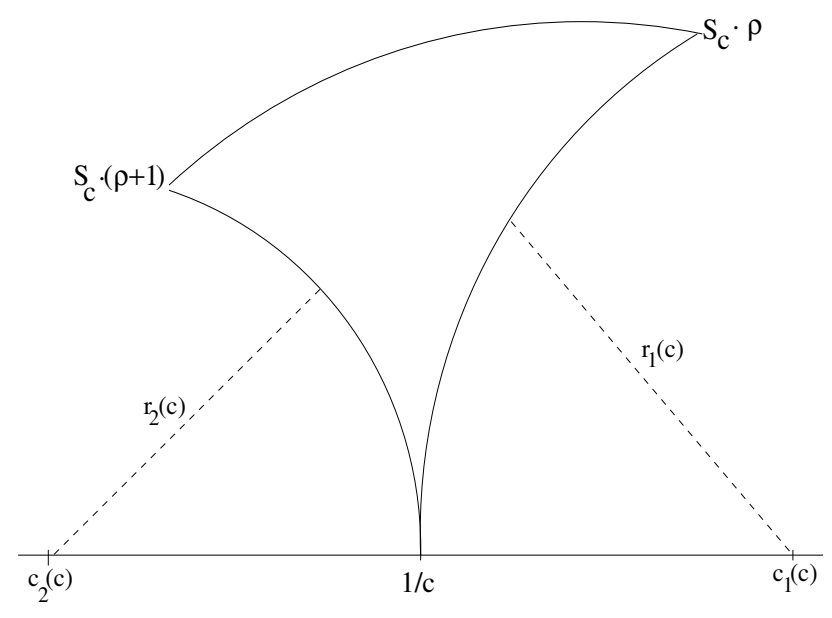

FiguRe 1

we have the following situation for the fundamental region $S_{c} \mathfrak{F}$ (see Figure 1).

The edge joining $1 / c$ and $S_{c} \cdot \rho$ is an arc of the circle $\mathcal{C}_{1}(c)$ centered at $c_{1}(c)=$ $(c-1) / c(c-2)$ and having radius $r_{1}(c)=1 / c(c-2)$, while the edge joining $1 / c$ and $S_{c} \cdot(\rho+1)$ is an arc of the circle $\mathcal{C}_{2}(c)$ centered at $c_{2}(c)=(c+1) / c(c+2)$ with radius $r_{2}(c)=1 / c(c+2)$. In particular, any other fundamental domain having the cusp $1 / c$ as a vertex is either within the circle $\mathcal{C}_{1}(c)$ or within the circle $\mathcal{C}_{2}(c)$.

The case $c=2$ needs to be clarified, as the radius $r_{1}(2)$ is infinite and in this case the arc joining $1 / 2$ and $S_{2} \cdot \rho$ is the vertical segment $[1 / 2,1 / 2+i \sqrt{3} / 6]$ (see Figure 2). Moreover, as we are restricting the study to the half-strip $\mathfrak{S}$, we only consider those fundamental domains with vertex at the cusp $1 / 2$ that lie under the arc of the circle $\mathcal{C}_{2}(2)$. It has center at $c_{2}(2)=3 / 10$ and radius $r_{2}(2)=1 / 10$.

Lemma 4.3. If we set

$$
M=\frac{1}{24}\left(1-E_{2}\left(\frac{i \sqrt{3}}{2}\right)\right),
$$

then we have

$$
24^{2}\left(M^{2}+\frac{M}{\pi}\right)<1
$$

Proof. Set $q=\exp (-\pi \sqrt{3})$. We have

$$
0<M=\sum_{n \geq 1} \sigma_{1}(n) q^{n}=\sum_{n \geq 1} \frac{n q^{n}}{1-q^{n}} \leq \frac{1}{1-q} \sum_{n \geq 1} n q^{n}=\frac{q}{(1-q)^{3}} .
$$

Hence, using (13), we have

Therefore,

$$
M \leq \frac{40000}{7880599}
$$

$$
24^{2}\left(M^{2}+\frac{M}{\pi}\right)<24^{2}\left(M^{2}+\frac{M}{3}\right) \leq \frac{61444600320000}{62103840598801}<1 .
$$




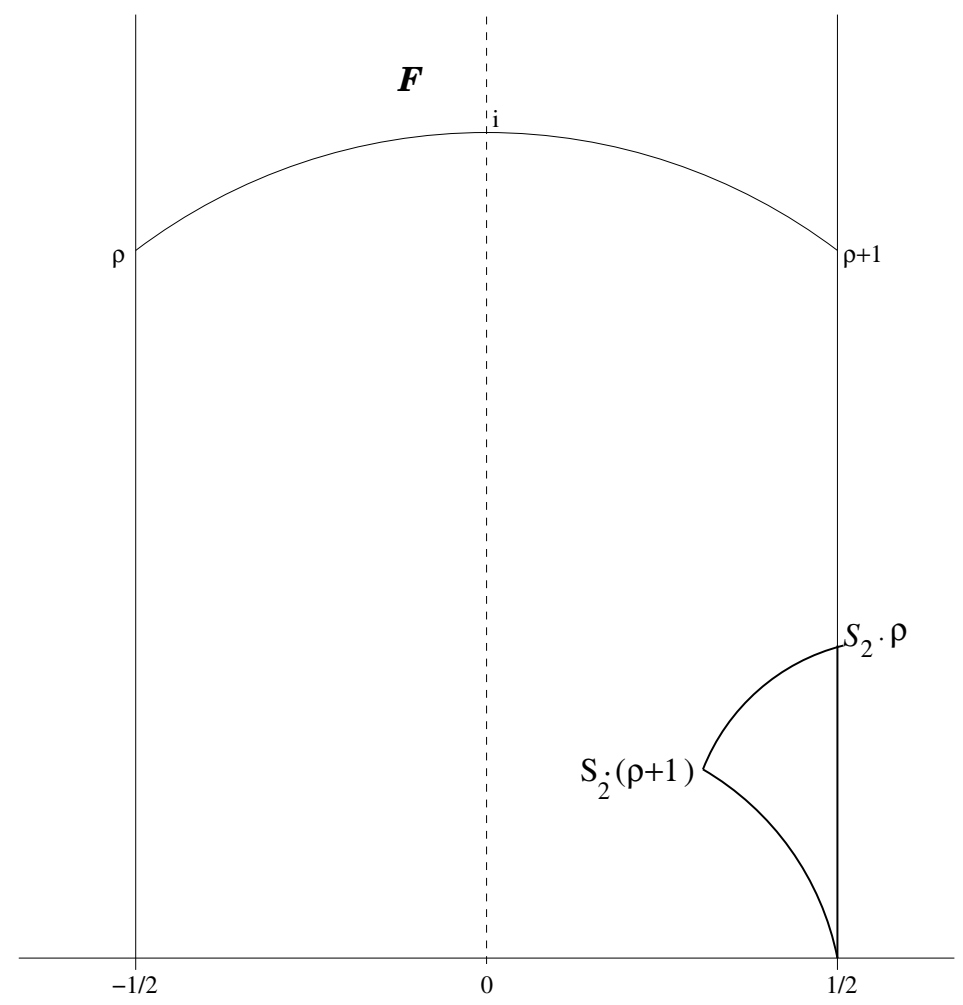

FiguRE 2

In the following, we will prove that the only fundamental domains having a vertex at the cusp $1 / c$ that might contain a zero of $E_{2}$ are the $\beta_{c} \mathfrak{F}$, and the only fundamental domain having a vertex at the cusp 0 that might contain a zero is $S \mathfrak{F}$.

Theorem 4.4. If $b \neq 0$, then $E_{2}$ has no zeros in $S_{b, d}(c) \mathfrak{F}$ or in $\delta_{b} \mathfrak{F}$.

Proof. Suppose first that $c \geq 3$, and suppose there is a zero $z_{0}$ of $E_{2}$ in the fundamental domain $S_{b, d}(c) \mathfrak{F}$ where $S_{b, d}(c)=\left(\begin{array}{ll}1 & b \\ c & d\end{array}\right) \in \mathrm{SL}_{2}(\mathbb{Z})$. If $b \neq 0$, then, according to the discussion preceding the above lemma, the fundamental domain $S_{b, d}(c) \mathfrak{F}$ is either within the circle $\mathcal{C}_{1}(c)$ or $\mathcal{C}_{2}(c)$. We will show that in fact $z_{0}$ is outside the circles $\mathcal{C}_{1}(c)$ and $\mathcal{C}_{2}(c)$, which is a contradiction.

We have

$$
E_{2}\left(S_{b, d}(c)^{-1} \cdot z_{0}\right)=\frac{-6 c}{\pi i}\left(-c z_{0}+1\right)
$$

so that

$$
\begin{aligned}
\sum_{n=1}^{\infty} \sigma_{1}(n) e^{2 \pi n i S_{b, d}(c)^{-1} \cdot z_{0}} & =\frac{1}{24}+\frac{c}{4 \pi i}\left(-c z_{0}+1\right) \\
& =-\frac{c^{2}}{4 \pi i}\left(z_{0}-\left(\frac{1}{c}+\frac{\pi i}{6 c^{2}}\right)\right) .
\end{aligned}
$$


Since $S_{b, d}(c)^{-1} \cdot z_{0} \in \mathfrak{F}$, we have

$$
\operatorname{Im}\left(S_{b, d}(c)^{-1} \cdot z_{0}\right) \geq \frac{\sqrt{3}}{2} .
$$

Hence

$$
\left|\sum_{n=1}^{\infty} \sigma_{1}(n) e^{2 \pi n i S_{b, d}^{-1} \cdot z_{0}}\right| \leq \sum_{n=1}^{\infty} \sigma_{1}(n) e^{-n \pi \sqrt{3}}=M .
$$

Therefore

$$
\left|z_{0}-\left(\frac{1}{c}+\frac{\pi i}{6 c^{2}}\right)\right| \leq M \frac{4 \pi}{c^{2}}
$$

that is, $z_{0}$ belongs to the disk $\mathcal{D}_{0}(c)$ of center $c_{0}(c)=\frac{1}{c}+\frac{\pi i}{6 c^{2}}$ and radius $r_{0}(c)=$ $M \frac{4 \pi}{c^{2}}$. We will now show that the disk $\mathcal{D}_{0}(c)$ lies outside the circles $\mathcal{C}_{1}(c)$ and $\mathcal{C}_{2}(c)$ by showing respectively that $\left|c_{0}(c)-c_{1}(c)\right|>r_{1}(c)+r_{0}(c)$ and that $\left|c_{0}(c)-c_{2}(c)\right|>$ $r_{2}(c)+r_{0}(c)$. Because the cusp $1 / c$ and $c_{0}(c)$ are on the same vertical axis, we have

$$
\left|c_{1}(c)-c_{0}(c)\right|^{2}=r_{1}(c)^{2}+\left(\frac{\pi}{6 c^{2}}\right)^{2} .
$$

Thus in order to prove that $\left|c_{0}(c)-c_{1}(c)\right|>r_{0}(c)+r_{1}(c)$ we only need to prove that

In other words,

$$
r_{0}(c)^{2}+2 r_{0}(c) r_{1}(c)<\left(\frac{\pi}{6 c^{2}}\right)^{2} .
$$

$$
2 \pi M^{2}+\frac{M c}{c-2}<\frac{\pi}{288} .
$$

In the meantime, for $c \geq 4$, we have $c /(c-2)=1+2 /(c-2) \leq 2$. Thus it is enough to prove that $2 \pi M^{2}+2 M<\pi / 288$, which is a consequence of Lemma 4.3.

Similarly, we prove that $\left|c_{2}-c_{0}\right|>r_{2}+r_{0}$. Indeed, as above, it is enough to show that

$$
2 \pi M^{2}+\frac{M c}{c+2}<\frac{\pi}{288}
$$

which is a consequence of Lemma 4.3 since $c /(c+2)<1$. Notice that $\left|c_{2}-c_{0}\right|>$ $r_{2}+r_{0}$ is also valid for the cases $c=2$ and $c=3$. This proves the theorem for $c \geq 4$ and also for $c=2$ since the circle $\mathcal{C}_{1}(c)$ is the vertical line $\operatorname{Re} z=1 / 2$, and thus we only need to estimate the distance $\left|c_{2}-c_{0}\right|$.

The case $c=3$ involves different estimates since we cannot apply Lemma 4.3 for the above choice of $M$. As we noticed above $z_{0}$ is outside the circle $\mathcal{C}_{2}(3)$, and we only need to show that it is outside $\mathcal{C}_{1}(3)$. On the other hand, the fundamental domain $S_{-1,-2}(3) \mathfrak{F}$ is adjacent (on the right) to $S_{3} \mathfrak{F}$ (see Figure 3 ), and the disc $\mathcal{D}_{0}(3)$ is outside the circle $\mathcal{C}_{3}$ which joins the vertices $1 / 3$ and $S_{-1,-2}(3) \cdot \rho$. Indeed, this circle is centered at $8 / 21$ and has radius $1 / 21$. Moreover

$$
\left|c_{0}(3)-8 / 21\right|=\frac{\sqrt{324+49 \pi^{2}}}{378} \approx 0.07518
$$

and

$$
r_{0}(3)+\frac{1}{21}=\frac{4 \pi M}{9}+\frac{1}{21}<\frac{4 \pi}{9 \cdot 200}+\frac{1}{21} \approx 0.0546 .
$$

It follows that the only possible values of $(b, d)$ for which $S_{b, d} \mathfrak{F}$ might contain a zero are $(b, d)=(-1,-2)$ leading to $S_{-1,-2}(3) \mathfrak{F}$ and $(b, d)=(0,1)$ leading to $S(3) \mathfrak{F}$. We now show that $z_{0} \notin S_{-1,-2}(3) \mathfrak{F}$ by exhibiting a smaller disc $\mathcal{D}(3)$ containing $z_{0}$ 


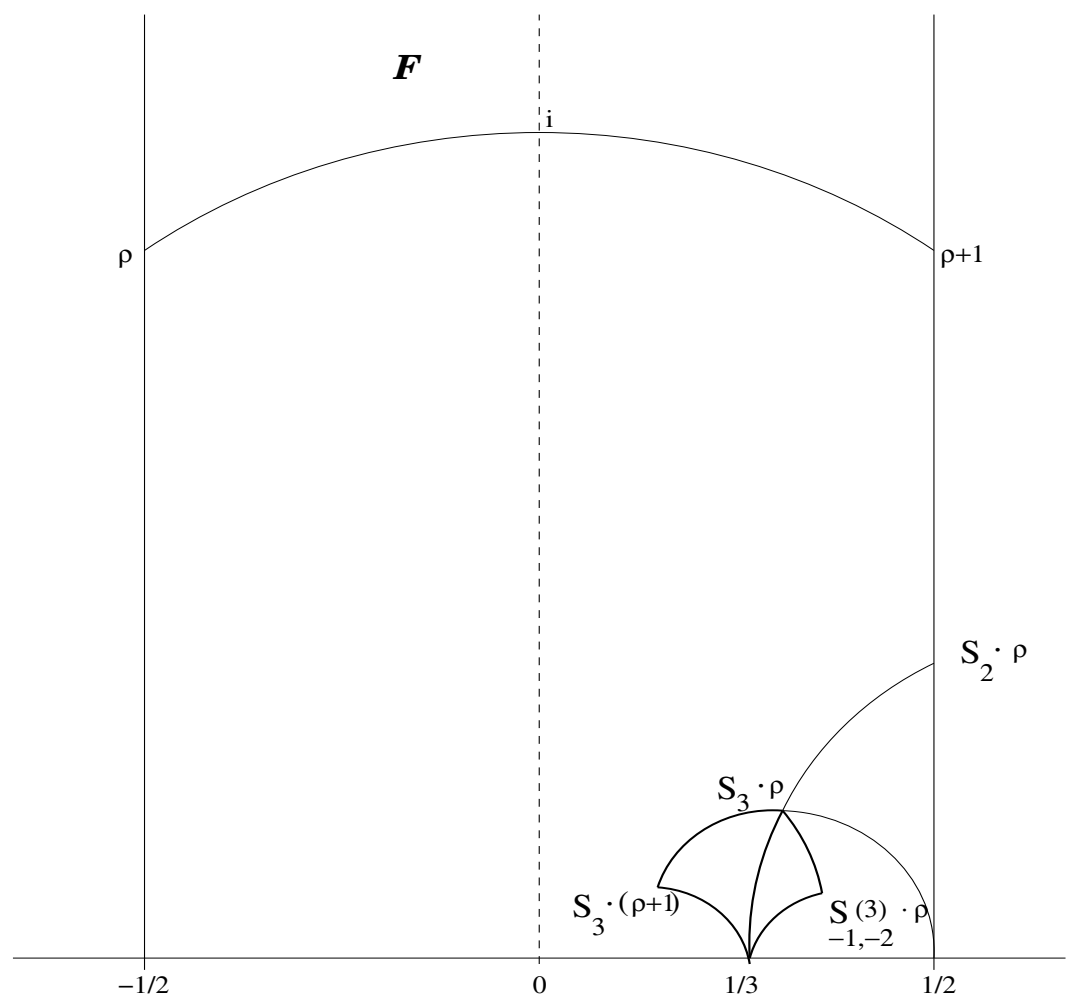

Figure 3

and lying outside the circle $\mathcal{C}_{1}(3)$ as the disc $\mathcal{D}_{0}(3)$ does not necessarily meet this condition. The transformation $S_{-1,-2}$ maps $\mathcal{D}_{0}(3)$ onto a disc $\mathcal{D}_{0}^{\prime}(3)$ centered at

$$
c_{0}^{\prime}(3)=S_{-1,-2}(3)^{-1} \cdot c_{0}(3)=\frac{6 i}{\pi}+\frac{2}{3}
$$

and with radius $r_{0}^{\prime}(3)$ that can easily be shown to satisfy $r_{0}^{\prime}(3)<0.26$. Therefore, we obtain a more precise lower bound to $\operatorname{Im} S_{-1,-2}(3) \cdot z_{0}$ as compared to (16):

$$
\operatorname{Im} S_{-1,-2}(3) \cdot z_{0}>\frac{6}{\pi}-0.26 \text {. }
$$

We now replace $M$ in Lemma 4.3 by

$$
M^{\prime}=\frac{1}{24}\left(1-E_{2}(i(6 / \pi-0.26))\right)
$$

and obtain

$$
2 \pi M^{\prime 2}+3 M^{\prime}<\pi / 288 .
$$

Hence, as in the general case, we conclude that

$$
\left|z_{0}-\left(\frac{1}{3}+\frac{i \pi}{54}\right)\right| \leq M^{\prime} \frac{4 \pi}{9}
$$

and therefore, the disc $D(3)=\mathcal{D}\left(1 / 3+i \pi / 54,4 \pi M^{\prime} / 9\right)$ is outside the circle $\mathcal{C}_{1}(3)$. It follows that there is no zero of $E_{2}$ in $S_{-1,-2}(3) \mathfrak{F}$ and thus in any $S_{b, d}(3) \mathfrak{F}$ for $b \neq 0$. 
Finally, for the case of the cusp at 0 , if $z_{0}$ is a zero of $E_{2}$ in $\delta_{b} \mathfrak{F}$, then $z_{0}$ is contained inside the circle centered at $\frac{\pi i}{6}$ and having radius $4 M \pi$ which is clearly contained in $S \mathfrak{F}$. Therefore $b=0$, since, otherwise, $\delta_{b} \mathfrak{F}$ and $S \mathfrak{F}$ are disjoint.

\section{REFERENCES}

[1] T. Falliero, A. Sebbar, Equilibrium point of Green's function for the annulus and Eisenstein series, Proc. Amer. Math. Soc. 135 (2007), no. 2, 313-328. MR2255277 (2007h:30011)

[2] S. Ramanujan, On certain arithmetical functions, Trans. Cambridge Philos. Soc. 22 (1916), no. 9, 159-184.

[3] R. A. Rankin, Modular forms and functions, Cambridge University Press, 1977. MR0498390 $(58: 16518)$

[4] R. A. Rankin, The zeros of certain Poincaré series, Compositio Math. 46 (1982), no. 3, 255272. MR664646 (83m:10036)

[5] F. K. C. Rankin, H. P. F. Swinnerton-Dyer, On the zeros of Eisenstein series, Bull. London Math. Soc. 2 (1970), 169-170. MR0260674 (41:5298)

[6] K. Wohlfahrt, Über die Nullstellen einiger Eisensteinreihen (German), Math. Nachr. 26 (1963/1964), 381-383. MR0167470(29:4743)

Department of Mathematics and Statistics, University of Ottawa, Ottawa, Ontario K1N 6N5, CANADA

E-mail address: asebbar@uottawa.ca

Department of Mathematics and Statistics, University of Ottawa, Ottawa, Ontario K1N 6N5, CANADA

E-mail address: aelba026@uottawa.ca 